ARTICLE

\title{
Proposed Weak Potential Generation in Creating Self-Mass of Neutrinos
}

\author{
HAO Lijuan ${ }^{1, *}$, Kenji ISHIBASHI ${ }^{1}$, Takeshi NISHIMURA ${ }^{2}$, Norichika TERAO $^{1}$, Hidehiko ARIMA $^{1}$ \\ ${ }^{1}$ Department of Applied Quantum Physics and Nuclear Engineering, Kyushu University \\ 744 Motooka, Nishi-ku, Fukuoka 819-0395 Japan \\ ${ }^{2}$ On leave to Mitsubishi Heavy Industries, Ltd., Kobe, Japan
}

\begin{abstract}
Neutrinos commonly exist in nature as a kind of lepton, and are known to have a quite small mass. The main purpose of this paper is to explain the generation of the self-mass of the neutrino through weak potential interaction. Neutrinos are treated to be composed of four types of constituent particles, and interaction potentials are produced by weak electric charge and weak electric dipole moment in Fermi gauge. The matrix properties of momentum introduce an equivalent time velocity in the potential generation in some cases. This velocity creates neither electric nor magnetic fields, and generates interaction energy with a scalar field $\hat{B}^{0}$. A formulation is given to define electromagnetic selfenergy of neutrino constituents. A calculation example is presented to explain the quite small self-mass of neutrino by the self-field energy in the weak interaction.
\end{abstract}

\section{KEYWORDS: neutrino, weak charge, weak dipole moment, neutrino structure, neutrino mass}

\section{Introduction}

Neutrinos commonly exist in nature as a kind of lepton. In the past decade, some neutrino oscillation experiments ${ }^{1)}$ have indicated that neutrinos own a very small mass. The experiment by our group ${ }^{2)}$ suggested that existence of an external scalar field $\hat{B}^{0}$ makes neutrino to be dissociated, which may also be the evidence of the small mass of neutrino. Electroweak theory, ${ }^{3,4)}$ which unified the electromagnetic and weak interaction, explained the mass of field bosons successfully. However, such theory was not applied to the study of neutrino structure. Until now, the neutrino structure and mass generation were not made clear.

A previous work of our group ${ }^{5)}$ gave a basic proposed structure of neutrino in terms of the weak-charge and weakdipole moment interaction. It described the motions of neutrino and its constituent particles. The mass of the neutrino was estimated without consideration of antisymmetrization of wave functions, and mass values were too large to be accepted.

In this paper, following the previous work, we attempt to explain the generation of the self-mass of the neutrino by revised potential calculations with the antisymmetrization. The self-mass is ascribed to the self-energy formed in the weak interaction, with which the kinetic mass should agree. Method of supplying the self-energy to neutrino should also be consistent to the kinetic-mass formulation mechanism.

We follow the Fermi gauge to define a scalar auxiliary field $\hat{B}^{0}$ which is useful to give a simple formulation of 4-vector potentials by d'Alembertian. This field may play a certain role in neutrino kinetic mass. The system having momentums of $\gamma^{0} \gamma^{\mu}$ and $\gamma^{0} \gamma^{5} \gamma^{\mu}$ matrices introduces reversed complex-type momentums, which work as a momentum in an equivalent time direction through the unit matrix equivalent to $\gamma^{0} \gamma^{0}$. We treat that the equivalent time momentum, i.e. velocity, generates $A^{0}$, and in turn, this field contributes to the self-mass of constituent particles of neutrino. Differing from the pre-

\footnotetext{
*Corresponding author, E-mail: haolijuan@kune2a.nucl.kyushu-u.ac.jp
}

(C)Atomic Energy Society of Japan vious work, in this paper, the interaction-energy-density between the scalar field $\hat{B}^{0}$ and equivalent time velocity will be excluded from the self-energy density in a form of Hamiltonian density.

\section{Kinetic Mass, $Q-Q_{d}$ Interaction, Subspace, and Matrix Properties}

A basic feature of assumptions ${ }^{6)}$ is briefly explained. A neutrino is assumed to consist of four types of constituent particles, according to the number of basic gamma matrices $\gamma_{v}$ with $v=0 \sim 3$. $^{7)}$ It is postulated that the constituent particles exist in either positive or negative mass states, i.e. $s_{v}^{m}= \pm 1$. The kinetic mass $m_{v}^{k i n}$ of internal neutrino constituent $v$ is defined as

$$
m_{v}^{k i n}=s_{v}^{m} \sqrt{\sum_{\mu}\left(p_{\mu, v V}\right)^{2}-\sum_{\mu}\left(p_{\mu, v A}\right)^{2}},
$$

where $p_{\mu, v V}$ and $p_{\mu, v A}$ are momentums of particle $v$ of vector(V)- and axial-vector(AV)-type motions in $\mu$ direction where $\mu=0 \sim 3$ indicates $c t, x, y$ and $z$.

We postulate that constituent particles possess either weak-
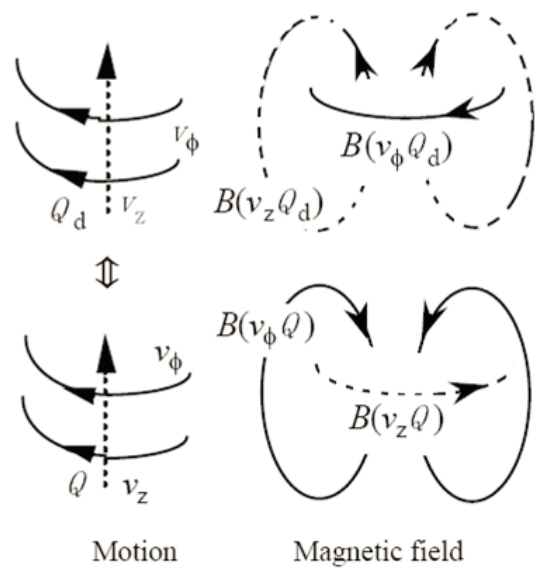

Fig. 1 Magnetic field generated by particles with charge $Q$ and dipole moment $Q_{d}{ }^{6}{ }^{6}$ The two particles are traveling in the $z$-direction with a left-rotated spiral motion. 


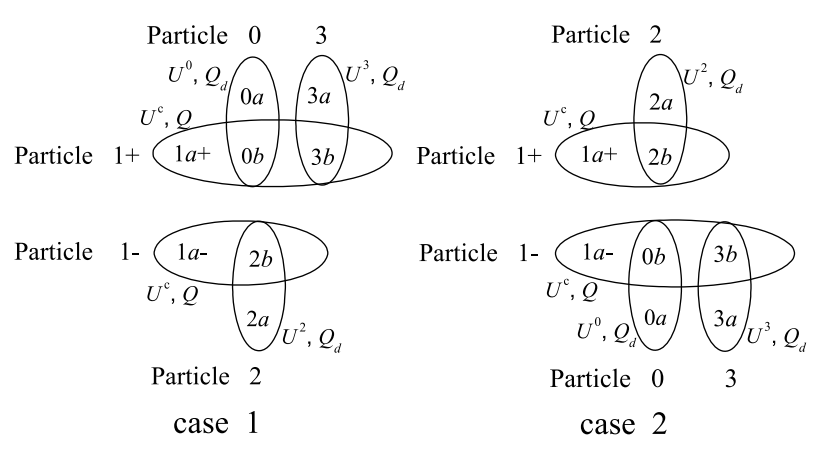

Fig. 2 Kinetic motions of constituent particles of neutrino described on common position of particle 1 . The position sharing facilitates the interaction in Fig. 1.

electric charge $Q$ or weak-electric-dipole moment $Q_{d}$. The dipole moment $Q_{d}$ is assumed to have a relationship to the charge: $Q_{d}=Q \hbar / p_{v}$, where $p_{v}$ is the momentum of interest. The magnetic fields between constituents particles are generated by $Q-Q_{d}$ pair, as illustrated in Fig. 1, where two particles having $Q$ and $Q_{d}$ are moving in the $z$-direction with a left-rotated spiral motion. From the figure, we can see that both the magnetic fields generated by $Q$ and $Q_{d}$ have individually opposite directions in principle. The two particles interact with each other to reduce magnetic-field energy, so that they tend to be in an equilibrium state.

In this study, we choose particle 1 to own electric charge $Q$, and to serve as the boson in the conventional space $U^{c}$ to supply the basic common motion. Quantities related to particle 1 itself are often specified by the use of $1 a$ thereafter. Other particles $v=0,2,3$ possess the dipole moment $Q_{d}$, and make their motion on a common position of particle 1 , as shown in Fig. 2. The transfer matrix $U_{v}$ from conventional $U^{c}$ system to relative systems (subspaces) $U^{v}$ is simply chosen as

$$
U_{v}=\left\{\begin{array}{ll}
\gamma_{v} & \text { for } v=0,1,3 \\
\gamma_{v} / i & \text { for } v=2
\end{array} .\right.
$$

Variables with subscript are used for indicating actual real values. Covariant properties ${ }^{8)}$ are expressed by superscripted variables, which include the imaginary unit $i$ in some cases. Defining superscripted values of gamma matrix $\gamma^{\mu}=\gamma_{\mu}$ for $\mu=0 \sim 3$, and $\gamma^{5}=\gamma_{5} / i$, where $\left(\gamma^{0} \gamma^{\mu}\right)^{2}=1$ and $\left(\gamma^{0} \gamma^{5} \gamma^{\mu}\right)^{2}=1$, we obtain the complex properties of variables, which are summarized in Table 1.

\section{Potential Generation and Propagation}

We consider that the $\mathrm{V}$ - and $\mathrm{AV}$-potentials propagate through the flight of $\mathrm{V}$ - and $\mathrm{AV}$-photons. The potentials are treated to travel in the space $U^{c}$. All positions and velocities (or momentums) are expressed in $U^{c}$ for the potential generation and propagation. Suppose that two particles $\rho$ and $\sigma$ exist at the same intrinsic time $\tau$, where $\rho$ takes a particle type in $1 a+, 1 a-, 0,2$ or 3 , and $\sigma$ also stands for one in those. When potentials propagate from the particle $\sigma$ at position $\left(x_{\sigma V}^{\mu}, x_{\sigma A}^{\mu}\right)$ to that of $\rho$ at $\left(x_{\rho V}^{\mu}, x_{\rho A}^{\mu}\right)$, the square of the difference of positions is written at the same intrinsic time $\tau$ by

$$
\left(x_{\rho V}^{0}-x_{\sigma V}^{0}\right)^{2}+\sum_{k=1 \sim 3}\left(x_{\rho V}^{k}-x_{\sigma V}^{k}\right)^{2}+\left(x_{\rho A}^{0}-x_{\sigma A}^{0}\right)^{2}+\sum_{k=1 \sim 3}\left(x_{\rho A}^{k}-x_{\sigma A}^{k}\right)^{2} .
$$

\begin{tabular}{|c|c|}
\hline $\mathrm{V}$ motion & AV motion \\
\hline$\left(\begin{array}{l}x_{v V}^{0} \\
x_{v V}^{k}\end{array}\right)=\left(\begin{array}{l}x_{0, v V} / i \\
x_{k, v V} / i\end{array}\right), \gamma^{0} \gamma^{\mu}$ & $\left(\begin{array}{c}x_{v A}^{0} \\
x_{v A}^{k}\end{array}\right)=\left(\begin{array}{l}x_{0, v A} \\
x_{k, v A}\end{array}\right), \quad \gamma^{0} \gamma^{\mu}$ \\
\hline$\left(\begin{array}{l}p_{v V}^{0} \\
p_{v V}^{k}\end{array}\right)=\left(\begin{array}{l}p_{0, v V} \\
p_{k, V V}\end{array}\right), \quad \gamma^{0} \gamma^{\mu}$ & $\left(\begin{array}{c}p_{v A}^{0} \\
p_{v A}^{k}\end{array}\right)=\left(\begin{array}{c}i p_{0, v A} \\
i p_{k, v A}\end{array}\right), \gamma^{0} \gamma^{5} \gamma^{\mu}$ \\
\hline$\left(\begin{array}{l}A_{v V}^{0} \\
A_{v V}^{k}\end{array}\right)=\left(\begin{array}{l}i A_{0, v V} \\
A_{k, v V}\end{array}\right), \quad \gamma^{0} \gamma^{\mu}$ & $\left(\begin{array}{l}A_{v A}^{0} \\
A_{v A}^{k}\end{array}\right)=\left(\begin{array}{l}A_{0, v A} \\
i A_{k, v A}\end{array}\right), \gamma^{0} \gamma^{5} \gamma^{\mu}$ \\
\hline$\hat{B}^{0}=-\sum \partial A^{\mu} / \partial x^{\mu}$, & $\hat{B}^{0}=-\sum \partial A^{\mu} / \partial x^{\mu}, \quad-\gamma^{5}$ \\
\hline
\end{tabular}

Table 1 Super/sub-scripted variables and matrix properties.

By the use of either retarded or advanced time $\tau^{\prime}=\tau+\varepsilon$, potentials arrive at the position $x_{\rho}$ at $\tau$ under the condition of $\left(x_{\rho V}^{0}-x_{\sigma V}^{\prime 0}\right)^{2}+\left(\boldsymbol{x}_{\rho V}^{k}-\boldsymbol{x}_{\sigma V}^{\prime k}\right)^{2}+\left(x_{\rho A}^{0}-x_{\sigma A}^{\prime 0}\right)^{2}+\left(\boldsymbol{x}_{\rho A}^{k}-\boldsymbol{x}_{\sigma A}^{\prime k}\right)^{2}=0$

where dashed values indicate the positions at $\tau^{\prime}$. Introduction of a spatial distance $d_{X}$ and a field-traveling time one $x_{X}^{0 f d}$ ( $X=V$ or $A$ ) changes the equation into

$d_{X} \equiv \sqrt{\left(x_{\rho X}-x_{\sigma X}^{\prime}\right)^{2}}=x_{\rho X}^{0 f d}-x_{\sigma X}^{\prime 0 f d}$

where

$$
\begin{aligned}
& x_{\rho X}^{0 f d}-x_{\sigma X}^{\prime 0 f d} \equiv \sqrt{-\left(x_{\rho V}^{0}-x_{\sigma V}^{\prime 0}\right)^{2}-\left(x_{\rho A}^{0}-x_{\sigma A}^{\prime 0}\right)^{2}-\left(x_{\rho X^{c}}-x_{\sigma X}^{\prime}\right)^{2}}, \\
& X^{c}=\left\{\begin{array}{l}
A \text { for } X=V \\
V \text { for } X=A
\end{array} .\right.
\end{aligned}
$$

In actual calculation, only increments $\partial x_{\rho X}^{0 f d}$ and $\partial x_{\sigma X}^{\prime 0 f d}$ are meaningful instead of $x_{\rho X}^{0 f d}$ and $x_{\sigma X}^{\prime 0 f d}$ themselves. Variation of the effective-time increment $\partial x_{\rho X}^{0 f d}$ at the arrival position is followed by the change in the potential generation position on the basis of eq.(3) as

$\left(d_{X}\right)^{-1}\left(\boldsymbol{x}_{\rho X}-\boldsymbol{x}_{\sigma X}^{\prime}\right) \cdot\left(-\partial \boldsymbol{x}_{\sigma X}^{\prime} / \partial x_{\sigma X}^{\prime 0 f d}\right) \partial x_{\sigma X}^{\prime 0 f d}=\partial x_{\rho X}^{0 f d}-\partial x_{\sigma X}^{\prime 0 f d}$. The relativistic time ratio $\partial x_{\sigma X}^{10 f d} / \partial x_{\rho X}^{0 f d}$ becomes to

$$
\frac{\partial x_{\sigma X}^{\prime 0 f d}}{\partial x_{\rho X}^{0 f d}}=\frac{1}{1-\left(d_{X}\right)^{-1}\left(\boldsymbol{x}_{\rho X}-\boldsymbol{x}_{\sigma X}^{\prime}\right)\left(\partial x_{\sigma X}^{\prime} / \partial x_{\sigma X}^{\prime 0 f d}\right)} .
$$

The potential propagating from the source to observation point needs to be multiplied by this ratio. When differentiation by $c \tau$ is expressed by "dot" on symbols, the value $\partial x_{\sigma X}^{\prime} / \partial x_{\sigma X}^{\prime 0 f d}$ leads to $\dot{\boldsymbol{x}}_{\sigma X}^{\prime} / \dot{x}_{\sigma X}^{\prime 0 f d}$, which stands for the spatial velocity of neutrino constituents with regards to the field-traveling time.

We follow the matrix-type definition in Table 1. For example, momentum $p_{v X}^{\mu}$, where $X=V$ or $A$, owns the matrix property of $\gamma^{0} \gamma^{\mu}$ for the V-type and $\gamma^{0} \gamma^{5} \gamma^{\mu}$ for AV-type. We take that the total weak charge of neutrino system equals the charge $e$ of electromagnetic interaction. For particle $v$, therefore, we adopt the charge $Q=e m_{v} / \sum_{v} m_{v}$, and the dipole moment $Q_{d}=Q \hbar / p_{v}$, where $\hbar / p_{v}$ is the de Broglie wave length on spatial momentum for particle $v$. In this study, the polarities of $Q$ and $Q_{d}$ are always taken to be fixed, and their variation in function is considered by means of apparent velocity $\beta_{v X}^{\mu}=p_{v X}^{\mu} /\left|m_{v}\right|$. The apparent velocity includes the mass information such as $s_{v}^{m} e^{i \zeta_{v A}}$, where $s_{v}^{m}$ is the mass polarity of \pm 1 and $e^{i \zeta_{v A}}$ indicates asymmetry ${ }^{6)}$ assumed for $U^{c}$ part ( $v b$ in Fig.2) of particle $v$. The charge and moment are considered as the scalar values. The matrix type of $A_{X}^{\mu}$ to be generated is treated as either $\gamma^{0} \gamma^{\mu}$, or $\gamma^{0} \gamma^{5} \gamma^{\mu}$ in accordance with the matrix property of momentum. 


\section{Potentials by $\boldsymbol{Q}_{d}$ motion}

The Lagrangian density $\tilde{L}_{X}$ is constructed at an intrinsic time $\tau$. The density $\tilde{L}_{X}$ is considered to have basically the same form for both $X=V$ and $A$. There is a difference in potential treatment between sources of $Q_{d}$ and $Q$. The basic part of the Lagrangian density $\tilde{L}_{\text {bas } X}$ is common for potential sources of $Q_{d}$ and $Q$ and described as

$$
\widetilde{L}_{\text {basX }}=\frac{1}{2 \mu_{0}}\left(\sum_{k=1 \sim 3} \frac{E_{X}^{k} E_{X}^{k}}{c}+\sum_{k=1 \sim 3} B_{X}^{k} B_{X}^{k}-\hat{B}_{X}^{0} \hat{B}_{X}^{0}\right)-\frac{1}{\mu_{0}} \hat{B}_{\mu=0 \sim 3}^{0} \sum_{\mu=} \frac{\partial A_{X}^{\mu}}{\partial x_{V X}^{\mu}},
$$

where simple subscripts are utilized here: $\left.\partial x_{v X}^{\mu}\right|_{\mu=k}=\partial x_{v X}^{k}$ and $\left.\partial x_{v X}^{\mu}\right|_{\mu=0}=\partial x_{v X}^{0 f d}$ as defined above. We adopt the Fermi gauge for introduction of the scalar auxiliary field $\hat{B}^{0}$ in the above Lagrangian density. The scalar field $\hat{B}^{0}$ acquires the meaning of the electric/magnetic-like field in the time direction. The potential generated by dipole moment $Q_{d}$ is described by the basic Lagrangian density minum interaction terms $V_{Q_{d} X}$ as

$$
\widetilde{L}_{Q_{d} X}=\widetilde{L}_{b a s X}-V_{Q_{d} X},
$$

where

$$
\begin{aligned}
& V_{Q_{d} X}=Q_{d X} \rho_{v X} c \hat{\beta}_{v X}^{0} \hat{B}_{X}^{0}+Q_{d X} \rho_{v X} c \sum_{k=1 \sim 3} \beta_{v X}^{k} B_{X}^{k}, \\
& Q_{d V}=Q_{d} / i, Q_{d A}=Q_{d}, \\
& \hat{\beta}_{v V}^{0}=0, \beta_{v V}^{k}=s_{v}^{m} \dot{x}_{v V}^{k}, \beta_{v A}^{k}=s_{v}^{m}\left(1-\delta_{k \kappa_{v}}\right) \dot{x}_{v a A}^{k}, \\
& \hat{\beta}_{v A}^{0}=\sqrt{\left(\dot{x}_{v A}^{\kappa_{v}}\right)^{2}+\chi_{v A}^{r e v} \sum_{k \neq \kappa_{v}}\left(\dot{x}_{v A}^{k}\right)^{2}}, \quad \chi_{v A}^{r e v}=\left\{\begin{array}{ll}
0 & \text { for } e^{i \zeta_{v A}=-1} \\
1 / 2 & \text { for } e^{i \zeta_{v A}=i}
\end{array} .\right.
\end{aligned}
$$

The symbol $\rho_{v X}$ stands for the spatial particle density, $\kappa_{v}=$ $0,3,2$ for particles $v=0,2,3$, respectively. ${ }^{6)}$ The symbolic expression of velocity is written as $\beta_{v X}^{k}=s_{v}^{m} \dot{x}_{v X}^{k}$. We discriminate $\beta_{v X}^{k}$ of normal complex type from $\beta_{v X}^{\kappa_{v}}$,rev having reversed complex type. $\left.{ }^{6}\right)$ When $\beta_{v X}^{\kappa_{v}, \text { rev }}$ appears, its root-squared-sum changes the matrix type into unit one through $\left(\gamma^{0} \gamma^{k}\right)^{2}=1$, and accordingly makes an equivalent normal complex type time velocity $\hat{\beta}_{v X}^{0}$. As in eq.(7), the product of $\hat{\beta}_{v X}^{0}$ and $\hat{B}_{A}^{0}$ generates the interaction energy in the equivalent time direction. For the V-type, $\beta_{v X}^{0}$ has no normal complex type value, and is incapable of producing potential in the time direction. It is noted that $\hat{\beta}_{v X}^{0}$ in the $\mathrm{AV}$-type retains the matrix of $\gamma^{5}$ as an exceptional treatment. That is, we consider that the rootsquared-sum operation deletes only the matrix properties of $\gamma^{0} \gamma^{k}$ with $\left(\gamma^{0} \gamma^{k}\right)^{2}=1$ in original matrix properties of $\gamma^{0} \gamma^{5} \gamma^{k}$ in $\beta_{v A}^{\kappa_{v}, \text { rev }}$ and $\gamma^{5}$ remains outside the root.

The conventional procedure on partial differentiation of the Lagrangian density defines the scalar field as

$$
\hat{B}_{X}^{0}=-\sum_{\mu=0 \sim 3} \frac{\partial A_{v X}^{\mu}}{\partial x_{v X}^{\mu}}-\mu_{0} Q_{d X} \rho_{v X} c \hat{\beta}_{v X}^{0} .
$$

Use of this relation simplifies the potential propagation equation. The time-component and spatial parts of 4-vector potentials are given by

$$
\left\{\begin{array}{l}
\square_{X} A_{X}^{0}=-\mu_{0} \frac{\partial}{\partial x_{v X}^{0}}\left(Q_{d X} \rho_{v X} c \hat{\beta}_{v X}^{0}\right), \\
\square_{X} \boldsymbol{A}_{X}=-\mu_{0} \nabla_{X} \times\left(Q_{d X} \rho_{v X} c \boldsymbol{\beta}_{v X}\right)-\mu_{0} \nabla_{X}\left(Q_{d X} \rho_{v X} c \hat{\beta}_{v X}^{0}\right),
\end{array}\right.
$$

where $\square_{X}$ is d'Alembertian working with position variables $x_{v X}^{\mu}$, and the rotation $\nabla_{X} \times$ and gradient $\nabla_{X}$ operators apply to variables of $x_{v X}^{k}$. It is noted that $x_{v X}^{0}$ in this context denotes $x_{v X}^{0 f d}$ in the potential propagation.

Through the function of magnetic moment, the spatial velocity $\boldsymbol{\beta}_{v X}$ in eq. (9) generates a regular vector potential component, which is written as $A_{X}^{k, \text { reg }}$. Besides this component, the velocity $\hat{\beta}_{v X}^{0}$ produces another potential component, i.e., $\hat{A}_{X}^{\mu}$ in $A_{X}^{\mu}$ with description of $A_{X}^{\mu}=A_{X}^{\mu, r e g}+\hat{A}_{X}^{\mu}$. Use of a scalar function $\hat{F}_{X}^{0}$ expresses $\hat{A}_{X}^{\mu}$ as

$$
\begin{aligned}
& \hat{A}_{X}^{\mu}=-\frac{\partial}{\partial x_{v X}^{\mu}} \hat{F}_{X}^{0}, \\
& \square_{X} \hat{F}_{X}^{0}=\mu_{0} Q_{d X} \rho_{v} c \hat{\beta}_{v X}^{0} .
\end{aligned}
$$

Since $\nabla_{X} \times\left(\nabla_{X} \hat{F}_{X}^{0}\right)=0$, it gives $\nabla_{X} \times \hat{\boldsymbol{A}}_{X}=\hat{\boldsymbol{B}}_{X}=0$ : $\hat{\boldsymbol{\beta}}_{v X}^{0}$ generates no magnetic field at all.

In addition, introduction of $\boldsymbol{F}_{X}^{\text {reg }}$ into $\boldsymbol{A}_{X}^{\text {reg }}$ description makes the calculation straight-forward as

$$
\begin{aligned}
& \boldsymbol{A}_{X}^{r e g}=\nabla \times \boldsymbol{F}_{X}^{r e g}, \\
& \square_{X} \boldsymbol{F}_{X}^{r e g}=-\mu_{0} Q_{d X} \rho_{v} c \boldsymbol{\beta}_{v X} .
\end{aligned}
$$

\section{Potentials by $Q$ motion}

The charge-type potential generation is applied on particle $1 a \pm$ of $\mathrm{V}$ and AV-type, and particle 2 of AV-type with opposite mass factor $e^{i \zeta_{V A}}=-1$ in $U^{c}$ motion ( $2 b$ in Fig.2). ${ }^{6}$ The Lagrangian density is written as

$$
\widetilde{L}_{Q X}=\widetilde{L}_{\text {basX }}-V_{Q X},
$$

with

$$
\begin{aligned}
& V_{Q X}=Q_{d X} \rho_{v X} c \hat{\beta}_{v X}^{0} \hat{B}_{X}^{0}+Q \rho_{v X} c \sum_{k=1 \sim 3} \beta_{v X}^{k} A_{X}^{k}, \\
& \hat{\beta}_{v V}^{0}=0, \beta_{v V}^{k}=\left\{\begin{array}{l}
s_{1 a}^{\pi} \dot{x}_{1 a V \pi}^{k} \\
s_{2}^{m} \dot{x}_{2 V}^{k}
\end{array}, \hat{\beta}_{v A}^{0}=\left\{\begin{array}{l}
\sqrt{\left(\dot{x}_{1 a A \pi}^{3}\right)^{2}} \\
\sqrt{\left(\dot{x}_{2 A}^{3}\right)^{2}}
\end{array},\right.\right. \\
& \beta_{v A}^{k}=\left\{\begin{array}{l}
s_{1 a}^{\pi} \beta_{1 A \pi}^{k} \\
s_{2}^{m}\left(1-\delta_{k 3}\right)\left(\chi_{2 A}^{n o r} \dot{x}_{3 A}^{k}\right)
\end{array}, \chi_{2 A}^{n o r}=-1 / 2,\right.
\end{aligned}
$$

where $\pi= \pm 1$ stands for particles $\pm 1 a$ and $\hat{\beta}_{v V}^{0}$ is set at zero for $\mathrm{V}$-motion. The scalar field and 4-vector potentials are again given by

$$
\hat{B}_{X}^{0}=-\sum_{\mu=0 \sim 3} \frac{\partial A_{X}^{\mu}}{\partial x_{v X}^{\mu}}-\mu_{0} Q_{d X} \rho_{v X} c \hat{\beta}_{v X}^{0},
$$

and

$$
\left\{\begin{array}{l}
\square_{X} A_{X}^{0}=-\mu_{0} \frac{\partial}{\partial x_{v X}^{0}}\left(Q_{d X} \rho_{v X} c \hat{\beta}_{v X}^{0}\right), \\
\square_{X} \boldsymbol{A}_{X}=-\mu_{0} Q \rho_{v X} c \boldsymbol{\beta}_{v X}-\mu_{0} \nabla_{X}\left(Q_{d X} \rho_{v X} c \hat{\beta}_{v X}^{0}\right) .
\end{array}\right.
$$

The V-motion of particle $1 a$ makes $A_{1 a V}^{0}=0$. Particle $1 a$ with charge $Q$ produces the scalar field $\hat{B}^{0}$ in both $\mathrm{V}$ - and $\mathrm{AV}$ types through eq. (14).

\section{Hamiltonian}

The form of Hamiltonian density $\widetilde{H}_{e m, X}$ for the weak electromagnetic field is derived from the Lagrangian density, although actual potential calculation is made by eqs. (8),(9), (14) and (15). The canonical conjugate momentums are expressed by 


$$
\pi_{X}^{0}=\frac{\partial L}{\partial\left(\partial A_{X}^{0} / \partial x_{X}^{0}\right)}=-\frac{\hat{B}_{X}^{0}}{\mu_{0}}, \quad \pi_{X}^{k}=\frac{\partial L}{\partial\left(\partial A_{X}^{k} / \partial x_{X}^{0}\right)}=\frac{1}{\mu_{0}} \frac{E_{X}^{k}}{c}
$$

where $X=V$ or $A$. These give the Hamiltonian density for dipole-moment and charge type interactions as

$$
\begin{aligned}
& \widetilde{H}_{e m, X}=\sum_{\mu=0 \sim 3} \pi_{X}^{\mu} \frac{\partial A_{X}^{\mu}}{\partial x_{X}^{0}}-\widetilde{L}_{e m, X} \\
& =\frac{1}{2 \mu_{0}}\left[\sum_{k=1 \sim 3} \frac{E_{X}^{k}}{c}\left(\frac{E_{X}^{k}}{c}+2 \frac{\partial A_{X}^{0}}{\partial x_{X}^{k}}\right)-\sum_{k=1 \sim 3} B_{X}^{k} B_{X}^{k}+\hat{B}_{X}^{0} \cdot\left(\hat{B}_{X}^{0}-2 \frac{\partial A_{X}^{0}}{\partial x_{X}^{0}}\right)\right] \\
& +\left[Q_{d X} \rho_{v X} c \hat{\beta}_{v X}^{0} \hat{B}_{X}^{0}+\left\{\begin{array}{l}
Q_{d X} \rho_{v X} c \sum_{k=1 \sim 3} \beta_{v X}^{k} B_{X}^{k} \\
Q \rho_{v X} c \sum_{k=1 \sim 3}^{k} \beta_{v X}^{k} A_{X}^{k}
\end{array}\right],\right.
\end{aligned}
$$

where the Hamiltonian density is composed of the fieldenergy-density and interaction-energy-density terms. The first one indicates the self-field energy density. The interaction between the scalar field $\hat{B}^{0}$ and equivalent time velocity $\hat{\beta}^{0}$ is included in the interaction-energy-density term for the logical consistency. This treatment is different from that in the previous work. ${ }^{5)}$ The spatial integral of the field-energy density on the particle location is considered to serve as the self-mass, and the kinetic motion of the neutrino constituents should take place to reproduce this self-mass.

\section{Test Calculation Result for Neutrino Mass}

Table 2 Calculation example of constituent mass in units of $\mathrm{eV}$ for neutrino total energy $1 \mathrm{MeV}$.

\begin{tabular}{crr}
\hline & \multicolumn{1}{c}{ case 1 } & \multicolumn{1}{c}{ case 2 } \\
\hline mass\#1 & $1.54 \times 10^{2}$ & $1.39 \times 10^{2}$ \\
mass\#0 & $3.65 \times 10^{2}$ & $-3.47 \times 10^{2}$ \\
mass\#2 & $-3.67 \times 10^{2}$ & $3.49 \times 10^{2}$ \\
mass\#3 & $2.00 \times 10^{0}$ & $-1.80 \times 10^{0}$ \\
total mass & $1.5 \times 10^{2}$ & $1.4 \times 10^{2}$ \\
\hline total mass(obs.) & $2.4 \times 10^{-2}$ & $1.9 \times 10^{-2}$ \\
\hline
\end{tabular}

The electromagnetic field energies in the particle region by eq. (17) serve as self-mass. A test calculation result of neutrino constituent mass is listed in Table 2, where the average radius of positive motion of particle $1 a$ was commonly set at a typical value of $10^{6} \mathrm{fm}$. Constituent mass values were searched by Monte Carlo method taking antisymmetrization of wave functions into consideration. The total kinetic mass is chosen to be the same as the weak-electromagnetic self-energy of the neutrino system. If individual-constituent masses in Table 2 are factored by the ratio of total mass to total energy, they are converted into the values in actual observation. The sum of converted values gives a total observed mass below eV level as shown in the lowest line in the table. General calculation with possibly less number of approximations will lead to a more realistic neutrino mass.

\section{Conclusion}

A neutrino is assumed to consist of four types of constituent particles, and interaction potentials are generated by weak charge and weak dipole moment in the Fermi gauge. The system has momentums with matrix property of $\gamma^{\gamma} \gamma^{\mu}$ and $\gamma^{0} \gamma^{5} \gamma^{\mu}$. When reversed complex-type velocities appear, they are converted into a value by root-squared-sum. The rootsquared-sum of $\gamma^{0} \gamma^{\mu}$ changes the matrix type into unit one, and make the equivalent time velocity $\hat{\beta}_{v X}^{0}$. This equivalent velocity generates no magnetic field at all, but the product of the velocity $\hat{\beta}_{v X}^{0}$ and scalar field $\hat{B}^{0}$ produces the interaction energy in the equivalent time direction. This interaction is excluded from the field-energy density, which should work as the self-energy to be consistent with the kinetic mass. A test calculation gave a neutrino mass below $\mathrm{eV}$ level.

\section{References}

1) For example, Q. R. Ahmad et al., “ Direct Evidence for Neutrino Flavor Transformation from Neutral-Current Interactions in the Sudbury Neutrino Observatory ", Phys. Rev. Letters, 89, 011301 (2002); K. Eguchi et al., " First Results from KamLAND: Evidence for Reactor Antineutrino Disappearance ", Phys. Rev. Letters, 90, 021802 (2003).

2) K.Ishibashi, Liu Wei et al., "Electrochemical neutrino detector and its signal generation scenario in relation to oxygen production", presented at this symposium.

3) S. Weinberg, "A model of leptons", Phys. Rev. Letters, 19, 1264 (1967) .

4) A. Salam, "Elementary Particle Theory" (N. Svartholom, Almquist and Forlag), 367 (1968).

5) T. Nishimura, K. Ishibashi, N.Terao, H. Arima,"Proposed structure of neutrino based on weak-charge and weak-dipolemoment interaction", Memoirs of the Faculty of Engineering, Kyushu University, 68, No.1 (2008).

6) K. Ishibashi et al., "Neutrino properties and their usefulness suggested by recent electrochemical neutrino detection results", presented at this symposium.

7) G. Kallen, "Quantum Electrodynamics", Springer-Verlag, Berlin (1972).

8) J. D. Bjorken, S. D. Drell, "Relativistic Quantum Fields", McGraw-Hill (1965). 